\title{
NUMERICAL ANALYSIS OF A RELAXED VARIATIONAL MODEL OF HYSTERESIS IN TWO-PHASE SOLIDS
}

\author{
Carsten Carstensen ${ }^{1}$ and Petr Plecháč ${ }^{2}$
}

\begin{abstract}
This paper presents the numerical analysis for a variational formulation of rate-independent phase transformations in elastic solids due to Mielke et al. The new model itself suggests an implicit time-discretization which is combined with the finite element method in space. A priori error estimates are established for the quasioptimal spatial approximation of the stress field within one time-step. A posteriori error estimates motivate an adaptive mesh-refining algorithm for efficient discretization. The proposed scheme enables numerical simulations which show that the model allows for hysteresis.
\end{abstract}

Mathematics Subject Classification. 65N30, 73C05.

Received: November 21, 2000. Revised: June 29, 2001.

\section{INTRODUCTION}

Typical features of phase transformations in solids include formation of fine phase mixtures in processes under which the material relaxes to an equilibrium configuration. In engineering applications, a typical size of the domain is several orders of magnitude larger than a characteristic length of the evolving microstructure. A numerical simulation of inhomogeneous boundary value problems therefore requires simplified phenomenological models. Here an averaging or relaxation procedure leads to a formulation (RIP) that can be interpreted as a macroscopic description of the material behavior. The original and the relaxed problem in the framework of single crystals of shape memory alloys are explored in $[1,2,15,17,21]$, their numerical analysis for the direct and relaxed minimization in $[8,9,14,18]$ and references quoted therein.

This paper focuses on the numerical analysis of a mathematical model due to Mielke et al. $[19,20]$ for the (quasistatic) evolution of phase transformations and hysteresis effects in crystalline solids. The resulting Relaxed Incremental Problem (RIP) reads: Given an initial phase mixture $\theta_{0}$ and a time-discretization $0<t_{1}<\cdots<$ $t_{J-1}<t_{J}=T$, seek mixtures $\theta_{j} \in \Xi^{* *}:=L^{2}\left(\Omega ;[0,1]^{N}\right), j=1,2, \ldots, J$, which solve the minimization problem

$$
\text { (RIP) } \quad \mathcal{I}^{r l x}\left(t_{j}, \theta_{j-1}, \theta_{j}\right)=\min _{\eta \in \Xi^{* *}} \mathcal{I}^{r l x}\left(t_{j}, \theta_{j-1}, \eta\right)
$$

Keywords and phrases. Variational problems, phase transitions, elasticity, hysteresis, a priori error estimates, a posteriori error estimates, adaptive algorithms, non-convex minimization, microstructure.

1 Institute for Applied Mathematics and Numerical Analysis, Vienna University of Technology, Wiedner Hauptstraße 8-10/115, A-1040 Wien, Austria. e-mail: Carsten.Carstensen@tuwien.ac.at

2 Mathematical Institute, University of Warwick, Coventry, UK. e-mail: plechac@maths.warwick.ac.uk 
Here, $\mathcal{I}^{r l x}(t, \theta, \eta)$ is the explicitly-known relaxation of the energy functional $\mathcal{I}(t, \xi, \zeta)=\mathcal{E}(t, \zeta)+\mathcal{D}(\xi, \zeta)$ at the time $t$ for the macroscopic mixtures $\xi, \zeta \in \Xi^{* *} ; \mathcal{E}(t, \zeta)$ is the free energy and $\mathcal{D}(\xi, \zeta)$ is the dissipation for the re-arrangement of $\xi$ into $\zeta \in \Xi^{* *}$ (see below).

The lowest order finite element method approximates the mixtures by $\mathcal{T}$-piecewise constants with respect to a regular triangulation $\mathcal{T}[4,10]$. The energy $\mathcal{E}(t, \xi)$ involves deformations which minimize energy; their lowest order finite element approximation employs continuous $\mathcal{T}$-piecewise affine finite elements. In each time step $j$, a discrete solution $\theta\left(t_{j}\right)$ of (RIP) exists but is not necessarily unique. Therefore, the error analysis faces severe difficulties caused by multiple solutions and the lack of uniform or even strict convexity. Nevertheless, we prove uniqueness and a quasioptimal error estimate for the exact and discrete stress field, establish a posteriori error control, and suggest an adaptive algorithm for efficient mesh-design. Control of the time-discretization error, however, lies beyond our analysis.

The paper and its main results are organized as follows. Section 2 provides a brief introduction into the variational model. The homogenization step from microstructure to a macroscopic description is performed in Section 3 on the level of mathematical relaxation of the energy functional. We provide explicit formulae for the energy densities in $\mathcal{I}^{r l x}$ in case of two (linearized) wells and equal elastic moduli. Section 4 introduces the spatial discretization and establishes uniqueness of the macroscopic stress and convergence of its discrete approximations. In contrast to a direct minimization [18], the discrete solution of the relaxed formulation here is not required to exhibit rapid oscillations to decrease the energy. Therefore, a numerical computation of discrete minimizers is feasible with sufficient accuracy and quasioptimal a priori stress error estimates can be derived. Based on the a posteriori stress error estimate of Section 5, we suggest an adaptive algorithm for automatic mesh-refinement. Numerical examples in Section 6 establish hysteresis as a typical feature of the numerical (hence of the mathematical) model.

\section{Mathematical MODEL}

This section introduces the mathematical model for a material body that occupies an open, connected, and bounded set $\Omega \subset \mathbb{R}^{d}(d=2,3)$ with the Lipschitz boundary $\Gamma:=\partial \Omega$ and its closed subset $\Gamma_{D} \subset \Gamma$ of positive Hausdorff measure, $\Gamma_{N}:=\Gamma \backslash \Gamma_{D}$. An equilibrium configuration consists of a fine mixture of $N$ phases, i.e., an $N$-tuple of characteristic functions

$$
\chi=\left(\chi^{(1)}, \ldots, \chi^{(N)}\right) \in \Xi \subset L^{\infty}\left(\Omega ;\{0,1\}^{N}\right),
$$

where $\chi^{(j)}(x)=1$ if the phase $j$ is found at the material point $x$ and $\chi^{(j)}(x)=0$ otherwise. The $N$ phases differ by their transformation strains $E_{j} \in \mathbb{R}_{\mathrm{sym}}^{d \times d}$ at the energy $W_{j}^{0}$ which define the energy density (potentials) of the separate phase $W_{j}$,

$$
W_{j}(E)=\left\langle E-E_{j}, \mathbb{C}\left(E-E_{j}\right)\right\rangle+W_{j}^{0},
$$

with an (equal) elastic tensor $\mathbb{C}$. Here, $\langle E, F\rangle:=\sum_{j, k=1}^{d} E_{j k} F_{j k}$ denotes the standard Euclidean scalar product in $\mathbb{R}_{\mathrm{sym}}^{d \times d}$ and $\mathbb{R}_{\mathrm{sym}}^{d \times d}$ denotes the symmetric real $d \times d$ matrices. The energy density of a phase arrangement sums up to

$$
W_{\chi}(E):=\sum_{j=1}^{N} \chi^{(j)} W_{j}(E) \text { for } \chi \in \Xi \text { and } E \in \mathbb{R}_{\mathrm{sym}}^{d \times d} .
$$

Given a phase mixture $\chi$ and a displacement $v$ with the (linear) Green strain tensor $\varepsilon(v):=\left(\nabla v+\nabla v^{T}\right) / 2 \in$ $\mathbb{R}_{\mathrm{sym}}^{d \times d}$, the elastic energy reads

$$
\mathcal{E}(\chi, v):=\int_{\Omega} W_{\chi}(\varepsilon(v)) \mathrm{d} x .
$$


External (time-dependent) forces, at the time $t$, are described by

$$
\mathcal{L}(t, v)=-\int_{\Omega} f \cdot v \mathrm{~d} x-\int_{\Gamma_{N}} g \cdot v \mathrm{~d} \gamma \quad \text { for } v \in V
$$

with given $f \in L^{2}\left(\Omega ; \mathbb{R}^{d}\right), g \in L^{2}\left(\Gamma_{N} ; \mathbb{R}^{d}\right), \Gamma_{N}:=\Gamma \backslash \Gamma_{D}$ and, $u_{D} \in W^{1,2}\left(\Omega ; \mathbb{R}^{d}\right)$. Set

$$
\begin{aligned}
& V:=\left\{v \in W^{1,2}\left(\Omega ; \mathbb{R}^{d}\right): v=u_{D} \text { on } \Gamma_{D}\right\} \quad \text { and, for } N=2, \\
& \Xi:=\left\{\left(\chi^{(1)}, \chi^{(2)}\right) \in L^{\infty}\left(\Omega ;\{0,1\}^{2}\right): \chi^{(2)}=1-\chi^{(1)} \in\{0,1\}\right\} .
\end{aligned}
$$

(We use standard notation for Sobolev and Lebesgue spaces.) Then, the total free energy of the arrangement $\chi \in \Xi$ at a time $t$ is

$$
\mathcal{E}(t, \chi):=\inf _{v \in V}\left\{\int_{\Omega} \sum_{j=1}^{N} \chi^{(j)} W_{j}(\varepsilon(v)) \mathrm{d} x+\mathcal{L}(t, v)\right\} .
$$

This paper addresses the model of quasi-static phase transitions $[19,20]$ with dissipation from the rearrangement of the phase configuration $\chi$ to the actual arrangement $\zeta$. A change of the phase arrangement $\chi$ into $\zeta$ dissipates the energy

$$
\mathcal{D}(\chi, \zeta):=\int_{\Omega} D(\chi, \zeta) \mathrm{d} x:=\int_{\Omega} \kappa|\chi-\zeta| \mathrm{d} x
$$

The Lipschitz-continuous but non-smooth choice $D(\chi, \zeta)=\kappa|\chi-\zeta|$ for some $\kappa \geq 0$ results in a rate-independent model $[19,20]$ in contrast, e.g., to the (smoother) quadratic ansatz of [16].

The variational approach of $[19,20]$ is based on a stability assumption: An arrangement $\zeta$ is called stable at a time $t$ if

$$
\mathcal{E}(t, \chi)+\mathcal{D}(\zeta, \chi) \geq \mathcal{E}(t, \zeta) \text { for all } \chi \in \Xi
$$

This maximum dissipation principle ensures that the system accommodates the minimum energy configuration at each point along the loading cycle. The hypothesis that the total energy achieves a global minimum at each time $t$ suggests a time evolution formulation.

Definition 2.1. (Incremental Problem). Given an initial phase arrangement $\chi_{0}$ and a time-discretization $0<$ $t_{1}<\cdots<t_{j-1}<t_{j}<\cdots<t_{J}=T$, seek a solution $\chi_{j} \in \Xi, j=1,2, \ldots, J$, of

$$
\mathcal{E}\left(t_{j}, \chi_{j}\right)+\mathcal{D}\left(\chi_{j-1}, \chi_{j}\right)=\inf _{\zeta \in \Xi}\left\{\mathcal{E}\left(t_{j}, \zeta\right)+\mathcal{D}\left(\chi_{j-1}, \zeta\right)\right\}
$$

The energy function $\mathcal{E}$ does not lead to a well-posed problem in the case of physically relevant forms of the functional $\mathcal{D}$ : The minimization problem (IP) may have no minimizers in the set of admissible arrangements $\Xi$. Hence, the problem (IP) is replaced by its relaxation (RIP) in the subsequent section.

\section{Relaxation of A two-Phase problem}

This section is devoted to explicit formulae for the relaxed problem (RIP) with $N=2$ wells. For a time $0<t \leq T$ and a mixture $\chi$ in $\Xi\left(\right.$ and below in $\left.\Xi^{* *} \subset L^{\infty}\left(\Omega ;[0,1]^{N}\right)\right)$ the energy $\mathcal{I}(t, \eta, \cdot)$,

$$
\mathcal{I}(t, \chi, \zeta):=\mathcal{E}(t, \zeta)+\mathcal{D}(\chi, \zeta)
$$

does not necessarily attain its infimum for $\zeta \in \Xi$ : The infimal energy

$$
\liminf _{k \rightarrow \infty} \mathcal{I}\left(t, \chi, \zeta_{k}\right) \text { for an infimising sequence }\left(\zeta_{k}\right) \stackrel{*}{\rightarrow} \zeta
$$


(with a weak limit $\zeta$ in $L^{\infty}\left(\Omega ;[0,1]^{N}\right)$ ) may not be attained in $\Xi$. Notice that, with $N=2$, the weak limit $\zeta$ belongs to

$$
\begin{aligned}
\Xi^{* *} & :=\left\{\left(\theta^{(1)}, \theta^{(2)}\right) \in L^{\infty}\left(\Omega ;[0,1]^{2}\right): 0 \leq \theta^{(2)}=1-\theta^{(1)} \leq 1\right\} \\
& =\left\{(\vartheta, 1-\vartheta): \vartheta \in L^{\infty}(\Omega ;[0,1])\right\} .
\end{aligned}
$$

The infimal energy obtained from an infimising sequence in (3) defines the lower semi-continuous envelope $\mathcal{I}^{r l x}(t, \eta, \cdot)$,

$$
\mathcal{I}^{r l x}(t, \eta, \zeta):=\inf _{\left(\zeta_{k}\right)^{*} \zeta} \liminf _{k \rightarrow \infty} \mathcal{I}\left(t, \eta, \zeta_{k}\right),
$$

where $\eta, \zeta \in \Xi^{* *}$ and the argument in the first infimum runs over all sequences $\left(\zeta_{k}\right)$ in $\Xi$ with the weak limit $\zeta$.

Definition 3.1. (Relaxed Incremental Problem). Given an initial $\theta_{0} \in \Xi^{* *}$ and a time-discretization $0<t_{1}<$ $\cdots<t_{j-1}<t_{j}<\cdots<T$

$$
\text { (RIP) minimize } \mathcal{I}^{r l x}\left(t, \theta_{j-1}, \theta_{j}\right) \text { for } \theta_{j} \in \Xi^{* *} \text {. }
$$

To describe the relaxed problem we define the quasiconvexification of $W_{\chi}$ at a fixed volume fraction.

Definition 3.2. For fixed $\theta=:(\vartheta, 1-\vartheta) \in \Xi$, we define the quasiconvexification of $W_{\chi}$ at the fixed volume fraction $\theta$ by

$$
W_{\theta}^{q c}(E)=\inf _{\chi} \inf _{v} \int_{(0,1)^{d}} W_{\chi}(E+\varepsilon(v)) \mathrm{d} x .
$$

In the infima, $\chi \in L^{\infty}\left((0,1)^{d} ;\{0,1\}^{2}\right), \chi=\left(\chi^{(1)}, 1-\chi^{(1)}\right)$ and

$$
\vartheta=\int_{(0,1)^{d}} \chi^{(1)} \mathrm{d} x,
$$

while the test displacement fields belong to $v \in C_{0}^{\infty}\left((0,1)^{d}\right)$.

The function $W_{\theta}^{q c}$ can be constructed explicitly.

Proposition 3.1. There exists $\gamma \geq 0$ such that, for any $\theta=:(\vartheta, 1-\vartheta) \in \Xi^{* *}$,

$$
W_{\theta}^{q c}(F)=\vartheta W_{1}(E)+(1-\vartheta) W_{2}(E)-\gamma \vartheta(1-\vartheta) \quad \text { with } E=\left(F+F^{T}\right) / 2 .
$$

Moreover, for $\eta, \theta \in \Xi^{* *}$, we have

$$
\mathcal{I}^{r l x}(t, \eta, \theta)=\inf _{v \in V}\left\{\int_{\Omega} W_{\theta}^{q c}(\nabla v) \mathrm{d} x+\mathcal{L}(t, v)\right\}+\int_{\Omega} \kappa|\eta-\theta| \mathrm{d} x .
$$

Proof. The formula for $W_{\theta}^{q c}(F)$ is due to [17] and $\gamma$ is defined therein. The general result on $\mathcal{I}^{r l x}$ is due to $[19,20]$ and hence we refer to their work for details.

The second part of the proposition is related to the concept of cross-quasiconvexity [11] and of $A$-quasiconvexity [13].

The numerical treatment of (RIP) in Algorithm 3.1 below exchanges the order of the two minimizations (in $W_{\theta}^{q c}(E)$ from Def. 3.2). The resulting problem involves an explicit representation of $\mathcal{I}^{r l x}$. The description and 
solution of which in Algorithm 3.1 requires the following formulae, where for each $E \in \mathbb{R}_{s y m}^{d \times d}$ and real $r$ and $s$,

$$
\begin{aligned}
& \ell(E)= \frac{1}{2 \gamma}\left(W_{2}(E)-W_{1}(E)\right)+\frac{1}{2}, \\
& H(r, s)= \begin{cases}\frac{\kappa}{2 \gamma} r & \text { if } s \leq-\frac{\kappa}{2 \gamma}, \\
\frac{\kappa}{2 \gamma} r-\frac{1}{2}\left(s+\frac{\kappa}{2 \gamma}\right)^{2} & \text { if }-\frac{\kappa}{2 \gamma} \leq s \leq r-\frac{\kappa}{2 \gamma}, \\
\frac{1}{2} r^{2}-r s & \text { if } r-\frac{\kappa}{2 \gamma} \leq s \leq r+\frac{\kappa}{2 \gamma}, \\
-\frac{\kappa}{2 \gamma} r-\frac{1}{2}\left(s-\frac{\kappa}{2 \gamma}\right)^{2} & \text { if } r+\frac{\kappa}{2 \gamma} \leq s \leq 1+\frac{\kappa}{2 \gamma}, \\
\frac{\kappa}{2 \gamma}(1-r)+\frac{1}{2}-s & \text { if } s \geq 1+\frac{\kappa}{2 \gamma},\end{cases} \\
& M(r, s)= \begin{cases}0 & \text { if } s \leq-\frac{\kappa}{2 \gamma}, \\
s+\frac{\kappa}{2 \gamma} & \text { if }-\frac{\kappa}{2 \gamma} \leq s \leq r-\frac{\kappa}{2 \gamma}, \\
r & \text { if } r-\frac{\kappa}{2 \gamma} \leq s \leq r+\frac{\kappa}{2 \gamma}, \\
s-\frac{\kappa}{2 \gamma} & \text { if } r+\frac{\kappa}{2 \gamma} \leq s \leq 1+\frac{\kappa}{2 \gamma}, \\
1 & \text { if } s \geq 1+\frac{\kappa}{2 \gamma} .\end{cases}
\end{aligned}
$$

A certain projection onto the space of symmetric matrices determines the constant $\gamma, 0<\gamma \leq \frac{1}{2}\left\langle E_{2}-E_{1}\right.$, $\left.\mathbb{C}\left(E_{2}-E_{1}\right)\right\rangle$. The two wells (transformation strains) $E_{1}$ and $E_{2}$ are called compatible, if

$$
E_{1}=E_{2}+\frac{1}{2}(a \otimes b+b \otimes a) \text { for some } a, b \in \mathbb{R}^{d} .
$$

In this case, $\gamma=\frac{1}{2}\left\langle E_{2}-E_{1}, \mathbb{C}\left(E_{2}-E_{1}\right)\right\rangle$ and $W_{\theta}^{q c}$ is a convex $C^{1}$ function; cf. [17] for details about two incompatible wells.

\section{Algorithm 3.1.}

(i) Compute a minimizer $u_{j} \in V$ of

$$
\min _{v \in V}\left\{\int_{\Omega}\left(W_{2}(\varepsilon(v))+2 \gamma H\left(\vartheta_{j-1}, \ell(\varepsilon(v))\right)\right) \mathrm{d} x+\mathcal{L}\left(t_{j}, v\right)\right\}
$$

(ii) Compute new arrangements $\theta_{j}=:\left(\vartheta_{j}, 1-\vartheta_{j}\right) \in \Xi^{* *}$ by

$$
\vartheta_{j}=M\left(\vartheta_{j-1}, \ell\left(\varepsilon\left(u_{j}\right)\right)\right) .
$$

The feasibility of Algorithm 3.1 and the next proposition imply existence of solutions of (RIP).

Proposition 3.2. Algorithm 3.1 computes a solution $\theta_{j}$ of (RIP).

Proof. The proof is a direct application of Proposition 3.1, the relaxation procedure of [17] and, for fixed $E$, a pointwise minimization of the function

$$
\vartheta W_{1}(E)+(1-\vartheta) W_{2}(E)-\gamma \vartheta(1-\vartheta)+\sqrt{2} \kappa\left|\eta^{(1)}-\vartheta\right| \text { over } 0 \leq \eta^{(1)} \leq 1 .
$$

We omit the details of a lengthy but elementary calculation.

\section{Finite Element approximation And CONVERGEnCE}

This section is devoted to the a priori error analysis of the spatial discretization of step (i) of Algorithm 3.1 and uniqueness of the exact and discrete stress variables. 
Let $V_{h} \subset V$ be a finite element space based on a triangulation $\mathcal{T}$ of the domain. Given $\theta_{j-1}$ and the energy $\mathcal{I}_{j}^{\text {rlx }}$ in step (i) of Algorithm 3.1,

$$
\mathcal{I}_{j}^{r l x}(v):=\int_{\Omega}\left(W_{2}(\varepsilon(v))+2 \gamma H\left(\vartheta_{j-1}, \ell(\varepsilon(v))\right)\right) d x+\mathcal{L}\left(t_{j}, v\right)
$$

suppose that $u \in V$ minimizes $\mathcal{I}_{j}^{r l x}$ in $V$ and $u_{h} \in V_{h}$ minimizes $\mathcal{I}_{j}^{r l x}$ in $V_{h}$. Then, let $\sigma(\varepsilon(u))$ and $\sigma_{h}:=\sigma\left(\varepsilon\left(u_{h}\right)\right)$ be the exact and discrete stress fields, respectively, where

$$
\sigma(E)=\mathbb{C}\left(E-E_{2}\right)-\partial_{s} H\left(\vartheta_{j-1}, \ell(E)\right) \mathbb{C}\left(E_{2}-E_{1}\right)
$$

The main a priori result of this paper states that the stress error converges quasioptimally in the energy norm.

Theorem 4.1. Suppose $E_{1}, E_{2} \in \mathbb{R}_{\text {sym }}^{d \times d}$ are compatible and that $u \in V$ and $u_{h} \in V_{h}$ are an exact and discrete minimizer of $\mathcal{I}_{j}^{r l x}$. Then,

$$
\left\|\mathbb{C}^{-1 / 2}\left(\sigma(\varepsilon(u))-\sigma\left(\varepsilon\left(u_{h}\right)\right)\right)\right\|_{L^{2}(\Omega)} \leq \min _{v_{h} \in V_{h}}\left\|\mathbb{C}^{1 / 2}\left(\varepsilon\left(u-v_{h}\right)\right)\right\|_{L^{2}(\Omega)} .
$$

\section{Remark 4.1.}

(a) Each of the continuous and the discrete stress fields is unique even though $u$ and $u_{h}$ may be non-unique. (This follows from the proof below.)

(b) Another interesting consequence of the arguments below is that $\sigma(\varepsilon(u))$ belongs to $W_{l o c}^{1,2}\left(\Omega ; \mathbb{R}_{\text {sym }}^{d \times d}\right)[3,7]$ but higher regularity results for $u$ cannot be expected ( $c f$. [22] for a similar situation).

(c) The right-hand side $\min _{v_{h} \in V_{h}}\left\|\mathbb{C}^{1 / 2}\left(\varepsilon\left(u-v_{h}\right)\right)\right\|_{L^{2}(\Omega)}$ in the theorem tends to zero as the mesh-size of the shape-regular triangulation tends to zero (by density). The convergence speed, though quasioptimal, cannot further be quantified as higher regularity of $u$ is not known.

(d) The multiplicative constant on the right-hand side in Theorem 4.1 is indeed 1.

(e) Note that $\theta_{j-1}$ is not generally known and so requires an approximation that involves an error from previous time-steps. Theorem 4.1 ignores this error source and merely analyses one single time-step.

(f) We failed to prove strong error estimates for

$$
\left|\theta-\theta_{h}\right|=\left|M\left(\vartheta_{j-1}, \ell(\varepsilon(u))\right)-M\left(\vartheta_{j-1}, \ell\left(\varepsilon\left(u_{h}\right)\right)\right)\right|
$$

of the step (ii) in Algorithm 3.1. As a consequence, there is no error analysis for the time-discretization available at the moment.

The proof given below is based on the following estimate.

Lemma 4.1. Suppose $E_{1}, E_{2} \in \mathbb{R}_{\text {sym }}^{d \times d}$ are two distinct compatible transformation strains and the relaxed stress field $\sigma(E)$ is defined as the derivative of $W_{2}(E)+2 \gamma H(\theta, \ell(E))$ with respect to $E$. Then we have (6) and the mapping $\sigma: \mathbb{R}_{\text {sym }}^{d \times d} \rightarrow \mathbb{R}_{\text {sym }}^{d \times d}$ is Lipschitz continuous and monotone with

$$
\left|\mathbb{C}^{-1 / 2}(\sigma(E)-\sigma(F))\right|^{2} \leq\langle\sigma(E)-\sigma(F),(E-F)\rangle .
$$

Proof. From the formulae of the previous section we obtain the stress field (6). Direct calculation shows that the function $g(s):=\partial_{s} H(r, s)$ is continuous and piecewise affine. Moreover the functions $g(s)$ and $f(s):=s+g(s)$ 
are monotone decreasing and increasing, respectively. Define $a:=\ell(E)$ and $b:=\ell(F)$ for two symmetric matrices $E, F$ and notice that $\left\langle(E-F), \mathbb{C}\left(E_{2}-E_{1}\right)\right\rangle=2 \gamma(b-a)$. Then we calculate,

$$
\begin{aligned}
& \left\langle\sigma(E)-\sigma(F), \mathbb{C}^{-1}(\sigma(E)-\sigma(F))\right\rangle-\langle E-F, \sigma(E)-\sigma(F)\rangle \\
& =\langle(\mathbb{C}(E-F)-(g(a)-g(b)) \mathbb{C}(E-F)),(g(a)-g(b))(E-F)\rangle \\
& =(g(b)-g(a))(2 \gamma(b-a)+2 \gamma(g(b)-g(a))) \\
& =2 \gamma(g(b)-g(a))(f(b)-f(a)) \leq 0 .
\end{aligned}
$$

Proof of Theorem 4.1. The continuous and discrete Euler-Lagrange equations and the definitions of $\sigma$ imply the Galerkin orthogonality

$$
\int_{\Omega}\left\langle\sigma(\varepsilon(u))-\sigma\left(\varepsilon\left(u_{h}\right)\right), \varepsilon\left(v_{h}\right)\right\rangle \mathrm{d} x=0 \quad \text { for each } v_{h} \in V_{h} .
$$

This, Lemma 4.1, and Cauchy inequality show, for $v_{h} \in V_{h}$, that

$$
\begin{aligned}
\int_{\Omega}\left|\mathbb{C}^{-1 / 2}\left(\sigma(\varepsilon(u))-\sigma\left(\varepsilon\left(u_{h}\right)\right)\right)\right|^{2} \mathrm{~d} x & \leq \int_{\Omega}\left\langle\sigma(\varepsilon(u))-\sigma\left(\varepsilon\left(u_{h}\right)\right), \varepsilon\left(u-u_{h}\right)\right\rangle \mathrm{d} x \\
& =\int_{\Omega}\left\langle\sigma(\varepsilon(u))-\sigma\left(\varepsilon\left(u_{h}\right)\right), \varepsilon\left(u-v_{h}\right)\right\rangle \mathrm{d} x \\
& \leq\left\|\mathbb{C}^{-1 / 2}\left(\sigma(\varepsilon(u))-\sigma\left(\varepsilon\left(u_{h}\right)\right)\right)\right\|_{L^{2}(\Omega)}\left\|\mathbb{C}^{1 / 2} \varepsilon\left(u-v_{h}\right)\right\|_{L^{2}(\Omega)} .
\end{aligned}
$$

\section{A POSTERIORI ERROR CONTROL AND ADAPTIVE MESH REFINEMENT}

This section is devoted to an a posteriori analysis for the stress error. Let $\Omega$ have a polyhedral boundary $\partial \Omega$ and let $\mathcal{T}$ be a regular triangulation of $\Omega$ into closed $d$-simplices in the sense of Ciarlet $[4,10]$. To simplify the notation, we restrict ourselves to triangles, parallelograms, tetrahedra, and parallelepipeds. The set of all faces in $\mathcal{T}$ is denoted as $\mathcal{F}$ while the skeleton $\Gamma_{\mathcal{F}}=\bigcup \mathcal{F}$ is the set of all elements' boundary points, and the boundary conditions do not change within one face.

Suppose that, under the conditions of Theorem 4.1, some discrete solution $u_{h} \in V_{h}$ and the discrete stress $\sigma_{h}:=\sigma\left(\varepsilon\left(u_{h}\right)\right)$ is known. Then, the volume and edge residuals $R \in L^{2}\left(\Omega ; \mathbb{R}^{d}\right)$ and $J \in L^{2}\left(\Gamma_{\mathcal{F}}, \mathbb{R}^{d}\right)$ are defined as residuals from the strong form of the Euler-Lagrange equation by

$$
\begin{aligned}
&\left.R\right|_{T}:=\left.\left(f+\operatorname{div} \sigma_{h}\right)\right|_{T} \quad \text { for } T \in \mathcal{T}, \\
&\left.J\right|_{E}:=\left\{\begin{array}{ll}
{\left[\sigma_{h} \cdot n_{E}\right]} & \text { if } E \not \subset \Gamma, \\
g-\sigma_{h} \cdot n & \text { if } E \subseteq \bar{\Gamma}_{N}, \\
0 & \text { if } E \subseteq \Gamma_{D},
\end{array} \text { for all } E \in \mathcal{F} .\right.
\end{aligned}
$$

We denote by $n_{E}$ a fixed unit normal vector along $E \in \mathcal{F}, n$ the outer normal unit vector on $\Gamma_{N}$, and the brackets [.] denote a jump of the discrete stress vectors $\sigma_{h} \cdot n_{E}$ across the inner face $E$. Let $h_{T}$ be the diameter of $T \in \mathcal{T}, h_{E}$ be the diameter of $E \in \mathcal{F}$, and set

$$
\begin{array}{rlrl}
h_{\mathcal{T}}: \bar{\Omega} \rightarrow(0, \infty), & \left.h_{\mathcal{T}}\right|_{T}=h_{T} & \text { for all } T \in \mathcal{T}, \\
h_{\mathcal{F}}: \Gamma_{\mathcal{F}} \rightarrow(0, \infty), & \left.h_{\mathcal{F}}\right|_{E} & =h_{E} & \text { for all } E \in \mathcal{F} .
\end{array}
$$


Theorem 5.1. Suppose $E_{1}, E_{2} \in \mathbb{R}_{\text {sym }}^{d \times d}$ are compatible and $\sigma, \sigma_{h}$ are an exact and discrete stress field. Then there exist positive constants $c_{1}$ and $c_{2}$, which depend only on the shape and not on the size of the elements in $\mathcal{T}$, such that, for all $v_{h} \in V_{h}$,

$$
\begin{aligned}
& \left\|\mathbb{C}^{-1 / 2}\left(\sigma(\varepsilon(u))-\sigma_{h}\right)\right\|_{L^{2}(\Omega)}^{2} \\
& \quad \leq\left\|\nabla\left(u-v_{h}\right)\right\|_{L^{2}(\Omega)}\left(c_{1}\left\|h_{\mathcal{T}} R\right\|_{L^{2}(\Omega)}+c_{2}\left\|h_{\mathcal{E}}^{1 / 2} J\right\|_{L^{2}\left(\Gamma_{\mathcal{F}}\right)}\right) .
\end{aligned}
$$

Proof. The proof is based on the identities in the proof of Theorem 4.1 and involves element-wise integration by parts, trace inequalities, and local approximation properties of quasi-interpolants; cf., e.g., [23] for details in a model problem. Since the arguments are related to those exploited in [9] for a similar application, we omit the details.

Theorem 5.1 motivates the automatic mesh-refinement scheme of Algorithm 5.1 (see, e.g., [12,23] for heuristic arguments for the design of adaptive schemes). On each element, we define

$$
\eta_{T}^{2}:=\left\|h_{\mathcal{T}} R\right\|_{L^{2}(T)}^{2}+\left\|h_{\mathcal{F}}^{1 / 2} J\right\|_{L^{2}(\partial T)}^{2}
$$

as the refinement indicator and calculate the global error estimator

$$
\eta^{2}:=\sum_{T \in \mathcal{T}} \eta_{T}^{2} .
$$

\section{Algorithm 5.1.}

(a) Start with a coarse mesh $\mathcal{T}^{k}, k=0$.

(b) Solve the discrete problem with respect to the actual mesh $\mathcal{T}^{k}$.

(c) Compute $\eta_{T}$ from (9) for all $T \in \mathcal{T}^{k}$.

(d) Evaluate global error estimator based on (10) and decide to terminate or to continue and then go to (e).

(e) Mark the element $T$ for (red) refinement provided

$$
\frac{1}{2} \max _{T^{\prime} \in \mathcal{T}^{k}} \eta_{T^{\prime}} \leq \eta_{T} .
$$

(f) Mark further elements (within a red-green-blue refinement) to avoid hanging nodes. Define the resulting mesh as the actual mesh $\mathcal{T}^{k+1}$, update $k$ and go to (b).

\section{Remark 5.1.}

(a) An upper bound for $\left\|\nabla\left(u-v_{h}\right)\right\|_{L^{2}(\Omega)}$ depends on the regularity (which is unknown; cf. [22] in a similar case).

(b) The upper bound of $\left\|\nabla\left(u-v_{h}\right)\right\|_{L^{2}(\Omega)} \leq\|\nabla u\|_{L^{2}(\Omega)}$ can be computed by growth conditions (and Korn's inequality) without the knowledge of higher regularity of the exact solution. This approach, however, leads to a huge overestimation by a factor $\sup _{v_{h} \in V_{h}}\|\nabla u\|_{L^{2}(\Omega)} /\left\|\nabla\left(u-v_{h}\right)\right\|_{L^{2}(\Omega)}$ which is expected of size $O(\sqrt{h})$. In the language of a posteriori error analysis, the estimate of Theorem 5.1 is reliable but not efficient.

(c) Estimates for the constants $c_{1}$ and $c_{2}$ may be found in [5]. For planar triangulations into right isoceles triangles (halved squares), we found $c_{1}, c_{2}<1$ in [6].

(d) For details on red-green-blue refinement strategies $c f .$, e.g., [23].

(e) The numerical experiments reported in the subsequent section employed a truncated Newton method within (each time step of) Algorithm 3.1. The conjugate gradient method with an ILU preconditioner computed the search directions from the sparse linear system of equations for the Hessian of the energy. This strategy allowed us to solve medium-size problems up to 40k unknowns. 


\section{NumericAl EXPERIMENTS}

This section reports on numerical experiments with two different materials (Materials A and B) and two different geometries (Case I and Case II). The two material phases have the same isotropic elasticity tensor $\mathbb{C}$, $\mathbb{C} E=\lambda(\operatorname{Tr} E) \operatorname{Id}+\mu(2 E-(\operatorname{Tr} E) \operatorname{Id}), \lambda=8.34, \mu=3.82$ transformation strains from Table 1 , and minimal energies $W_{1}^{0}=W_{2}^{0}=0$. The initial phase in Material $\mathrm{A}$ is $\theta_{0}^{(1)}=\theta_{0}^{(2)}=1 / 2$ and in Material $\mathrm{B}$ is $\theta_{0}^{(1)}=1$, $\theta_{0}^{(2)}=0$.

TABLE 1. Transformation Strains for Materials A and B.

\begin{tabular}{|l|c|c|}
\hline & Material A & $\begin{array}{c}\text { Material B } \\
(\mathrm{CuZnAl})\end{array}$ \\
\hline $\begin{array}{l}\text { Phase transformation } \\
\text { Compatibility }\end{array}$ & martensite-martensite & austenite-martensite \\
compatible & incompatible \\
Transformation strain $E_{1}$ & $\left(\begin{array}{cc}-0.1 & 0 \\
0 & 0.1\end{array}\right)$ & $\left(\begin{array}{cc}0 & 0 \\
0 & 0\end{array}\right)$ \\
Transformation strain $E_{2}$ & $\left(\begin{array}{cc}0.1 & 0 \\
0 & -0.1\end{array}\right)$ & $\left(\begin{array}{cc}0.045 & 0.02 \\
0.02 & 0.045\end{array}\right)$ \\
\hline
\end{tabular}

The unit square $\Omega=(0,1)^{2}$ is traction free on $\Gamma_{N}^{1} \cup \Gamma_{N}^{2}$ and considered with two different Dirichlet conditions on $\Gamma_{D}^{1} \cup \Gamma_{D}^{2}$ depicted in Figure 1. The hysteresis loop is induced by cyclic changes of the horizontal displacements $u_{1}=w(t):=0.05 t$ for $-3 \leq t \leq 3$ at $\Gamma_{D}^{2}$. Note that the case of displacement-driven experiment simulated in the presented computation is not completely covered by our analysis as the Dirichlet boundary condition changes at each time step.

The uniaxial tension test is modeled in Case I as the simplest experiment for hysteresis loops. Only the first component $u_{1}$ of the displacement field is fixed on $\Gamma_{D}^{1} \cup \Gamma_{D}^{2}$ while $u_{2}$ is completely free (the free vertical translation is fixed at the node $\left.(0,1 / 2) \in \Gamma_{D}^{1}\right)$.
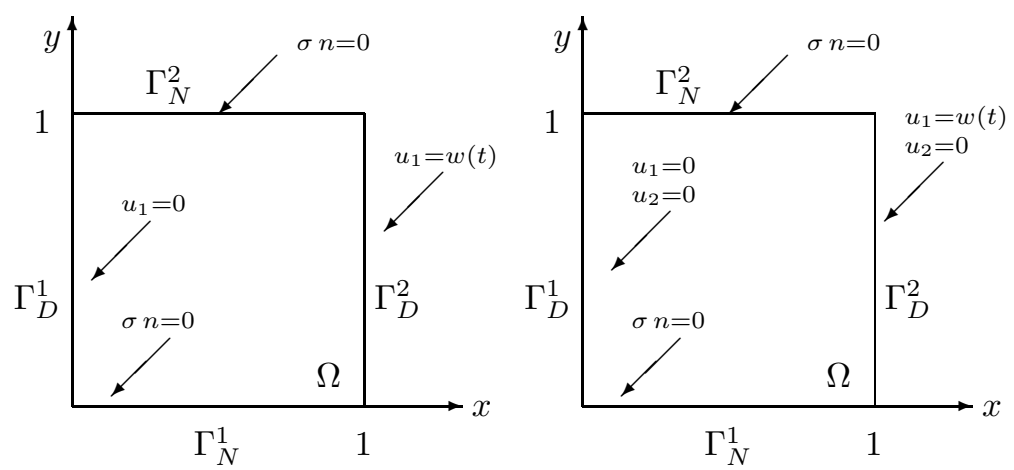

Figure 1. Configuration in Case I (left) and Case II (right).

The first set of numerical experiments concerns Case I with Material A for $\kappa=5$ and a coarse triangulation $\mathcal{T}_{0.1}$ of 400 triangles. For a positive integer $K, \mathcal{T}_{1 / K}$ is designed by a uniform partition $\Omega$ into $K \times K$ congruent squares (each of size $1 / K$ ) followed by a partition of each such square into 4 congruent triangles by criss-cross refinement (i.e., cutting the squares along the two diagonals).

The material deformed homogeneously (each square behaves in the same way) and so the coarse mesh was fixed while different time-steps $\Delta t$ were employed for a load in the cycle in which the time parameter $t$ was 

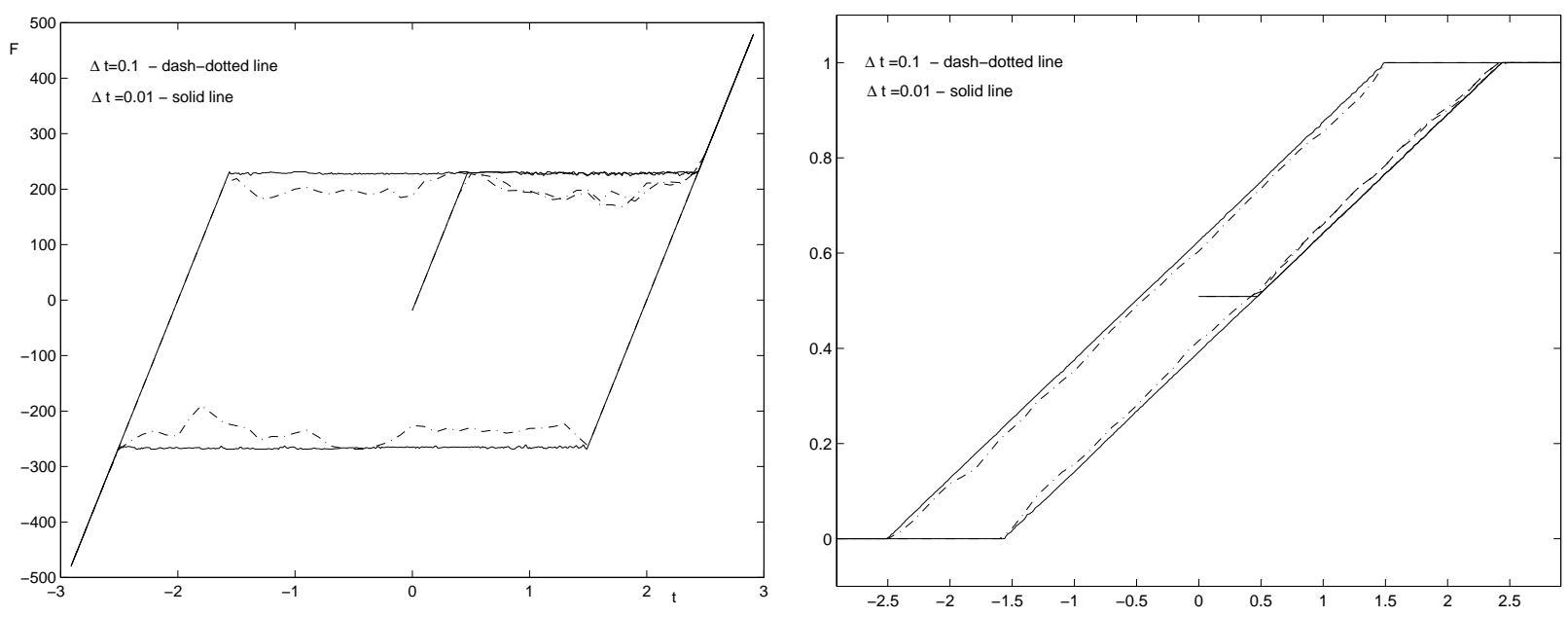

FiguRE 2. Load-displacement diagrams (left) and global volume fractions along the loop (right) for Material A in Case I.

monotonously moved from 0 up to 3 , then down to -3 and up to 3 again. The corresponding cycle is displayed in Figure 2 for $\Delta t=0.1$ and 0.01 where

$$
F(t)=\int_{\Gamma_{D}^{2}}\left(\sigma_{h}\right)_{11} \mathrm{~d} x \text { and } \Theta(t):=\int_{\Omega} \theta_{h} \mathrm{~d} x
$$

are plotted as a function of $t ; F(t)$ is the total force required to pull the specimen. We observe a typical hysteresis loop which appears smoother for finer time-discretization. The phase change is displayed by the total volume fraction $\Theta(t)$. Our numerical experiments show that cyclic stretching and compressing resulted in a stable plot.

The same Material A is subject to the different boundary conditions of Case II in the second set of numerical experiments to model a two-dimensional material behavior. The load-displacement diagram (left) and global volume fractions (right) along the loop is displayed in Figure 3 for different time-steps $\Delta t=0.1$ and 0.01 and different meshes $\mathcal{T}_{0.1}$ and $\mathcal{T}_{0.01}$ with mesh sizes $h=0.1$ and $h=0.01$. The curves show a rounded hysteresis loop which is qualitatively similar to that of Figure 2; the finer the discretization the smoother is the numerical approximation. Our interpretation is that the numerical scheme converges to the curved loop which results from two-dimensional inhomogeneous stress and strain fields.

Within the second set of experiments, we monitor the stress approximation and the need of local meshrefinement. Figure 4 displays the mesh $\mathcal{T}^{9}$ obtained at $t=0.51$ after 50 time-steps of Algorithm 3.1 with $\Delta t=0.01$ on a mesh $\mathcal{T}_{0.25}$ to yield a coarse approximation to $\theta_{50}$ and then by one run of Algorithm 5.1 (at another time step for $t=t_{51}$ ). The mesh $\mathcal{T}^{9}$ with 12800 elements is generated by Algorithm 5.1 in 9 refinement steps and shows a high refinement towards the corner points of the domain. Since the type of boundary conditions changes at these points, this is expected; high stress variations near the corner points of the right figure indicate necessary refinement there.

To give quantitative evidence of an improvement of the approximations, by adaptive mesh-refinements, we computed the energy and the error estimator for two runs of Algorithm 5.1 and compared the results in Figure 5.

The dashed line displays the energy $\mathcal{I}_{50}^{r l x}\left(u_{h}\right)-E_{0}\left(E_{0}=15.1078\right.$ is the minimal energy calculated by extrapolation) versus the number of unknowns $N$ for a uniform mesh-refinement by successive red-refinements of $\mathcal{T}^{0}=\mathcal{T}_{0.25}$. Convergence of the energies is seen in Figure 5 for a uniform and two adapted refinement sequences 

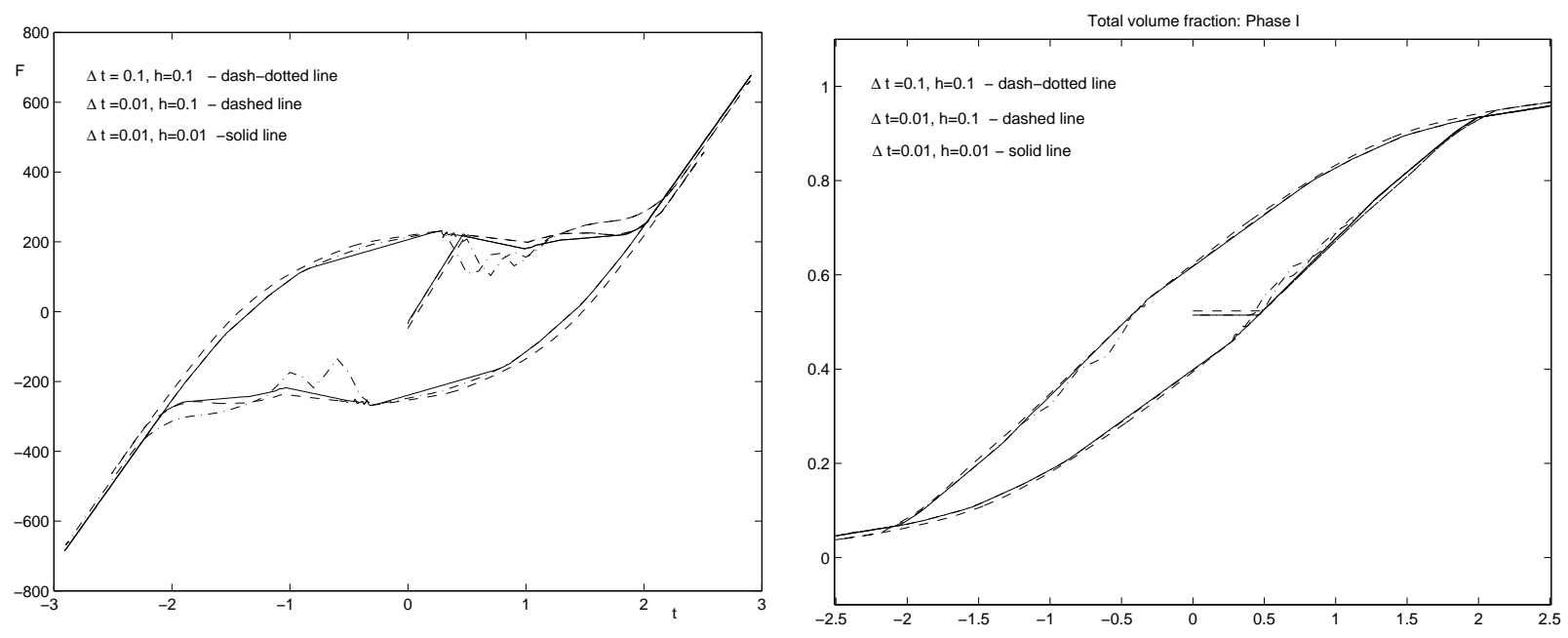

FiguRE 3. Load-displacement diagrams (left) and global volume fractions along the loop (right) for Material A in Case II.
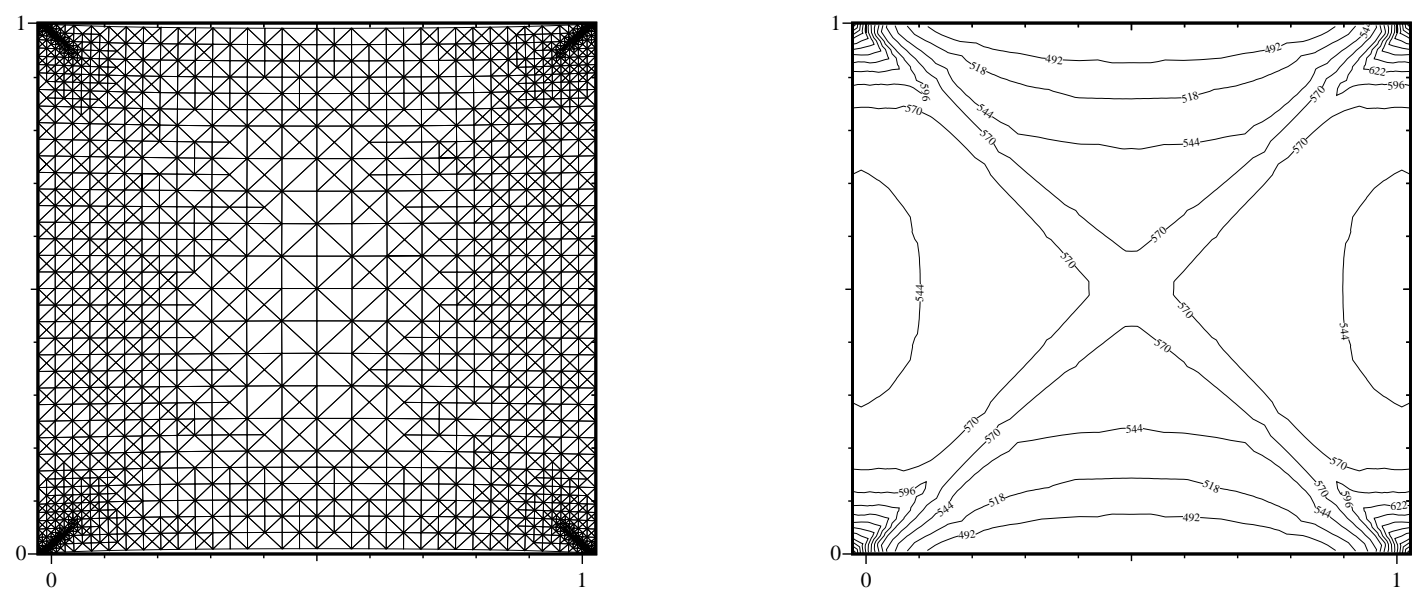

Figure 4. Mesh $\mathcal{T}^{9}$ generated by Algorithm 5.1 with 2993 unknowns (right) and contours of $\left|\sigma_{h}\right|$ on a fine mesh (right) for Material A in Case II.

with different coarse meshes $\left(\mathcal{T}^{0}=\mathcal{T}_{0.25}\right.$ with 64 elements resp. $\mathcal{T}^{0}=$ red-refinement of $\mathcal{T}_{0.25}$ with 252 elements). In both cases, the adaptive meshes lead to smaller energies but the convergence rate (of approximately $4 / 3$ ) for uniform meshes is not significantly improved.

This is not in contradiction to our theoretical results since our error estimates do not cover the energy. While the exact stress is unknown, the error estimator $\eta$ for the discrete stress $\sigma_{h}:=\sigma\left(\varepsilon\left(u_{h}\right)\right)$ is computable. Note carefully, that a reliable version of Theorem 5.1 states

$$
\left\|\mathbb{C}^{-1 / 2}\left(\sigma(\varepsilon(u))-\sigma_{h}\right)\right\|_{L^{2}(\Omega)} \leq C \sqrt{\eta}
$$



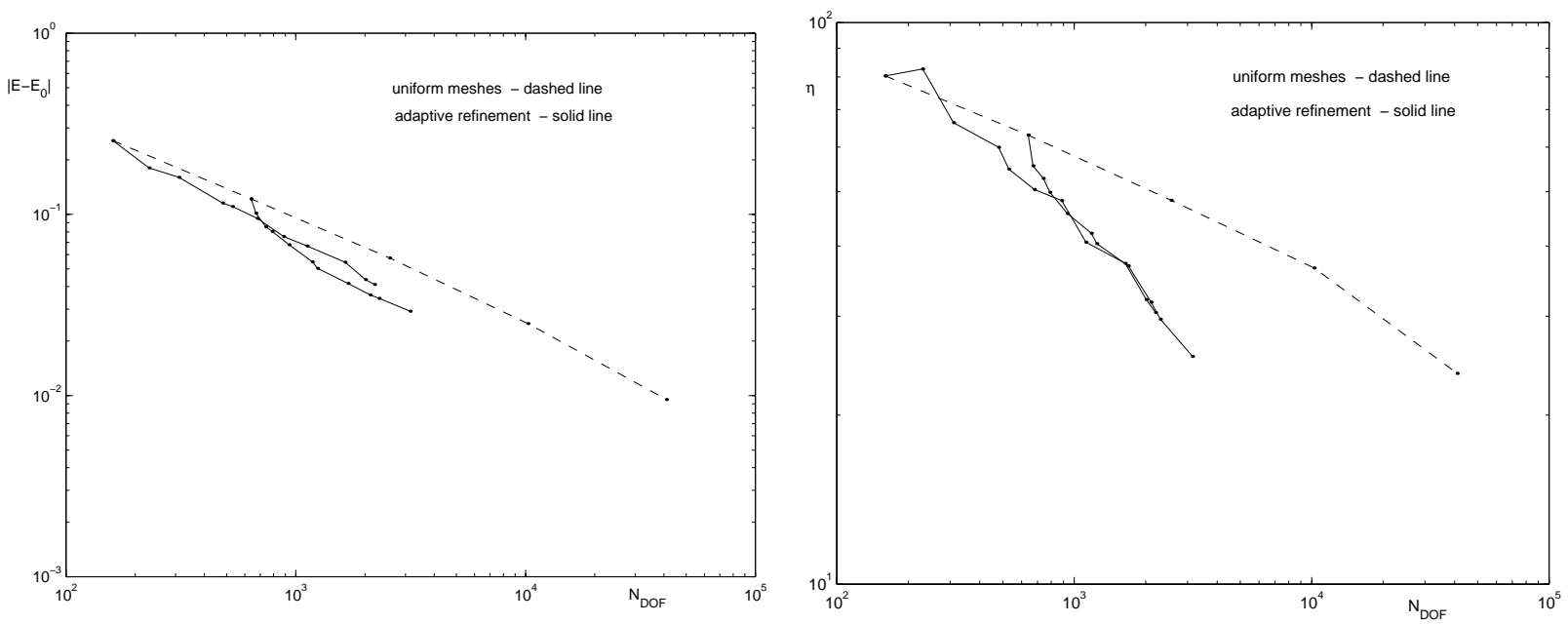

Figure 5. Convergence of the energy $\mathcal{I}_{j}^{r l x}$ (left) and of a posteriori error indicator $\eta$ (right) for adaptively refined meshes and uniform meshes at $t=0.51$ for Material A in Case II.

while the right plot of Figure 5 gives $\eta$ versus $N$. Hence the experimental convergence rate about $1 / 3$ of $\eta$ for the uniform (red) mesh-refinements corresponds to a poor guaranteed convergence of $1 / 6$. The adaptive meshes show a much better behavior close to the optimal linear convergence for $\eta$.

This might be regarded as an experimental support for an improvement of the adaptive mesh-generation over a naive uniform refinement at least for the upper stress-error bound $\eta$.

In the third series of numerical experiments, the Material B of Table 1 with $\kappa=10$ is considered in Case I. Note that the undeformed body is in equilibrium for around $t=0$ and so the starting point is already part of the hysteresis loop.
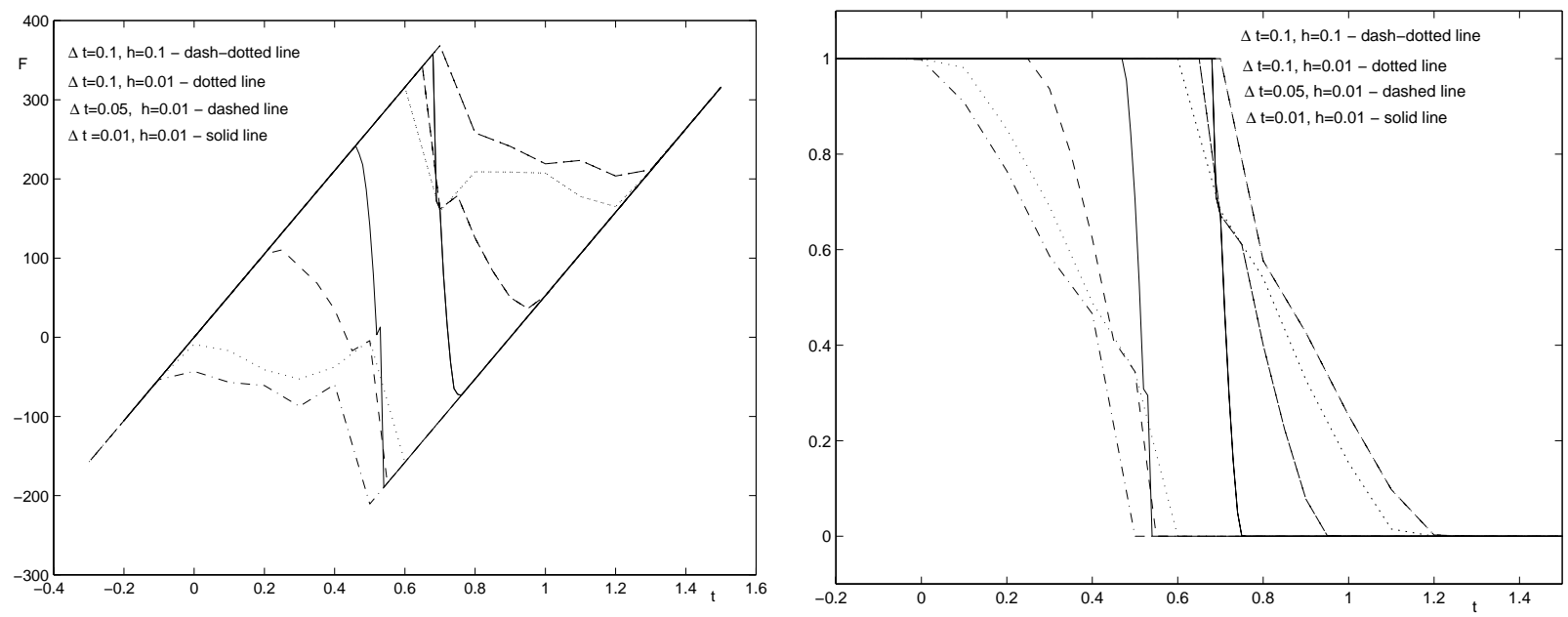

FiguRE 6. Load-displacement diagrams (left) and global volume fractions along the loop (right) for Material B in Case I. 
Since the transformation strains are rotated against the symmetry axis of the domain, this situation is not an uniaxial case, and, in contrast to Material A, the mechanical response is unsymmetric. Figure 6 shows load-displacement diagrams (left) and global volume fractions along the loop for different time-steps $\Delta t=0.1$, 0.05, and 0.01 and different meshes $\mathcal{T}_{0.1}$ and $\mathcal{T}_{0.01}$ as before. Several numerical tests convinced us that the hysteresis loop is stable and can be reproduced many times. For different meshes and time-steps, however, the hysteresis loop appears very different.

The choice of transformation strains corresponds to an incompatible austenite-martensite transformation. Therefore, the theoretical estimates of this paper are not applicable; our numerical experiments may support convergence of the scheme (in time and space) for this example.
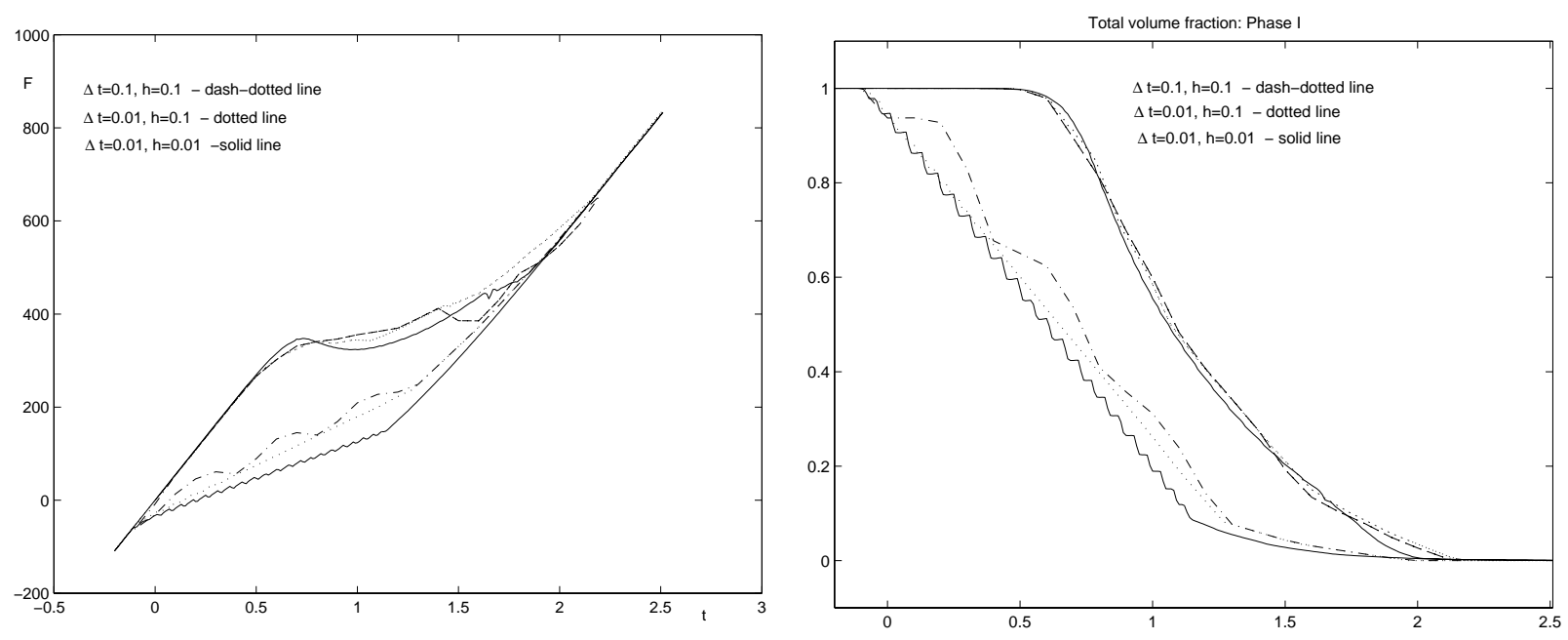

FigURE 7. Load-displacement diagrams (left) and global volume fractions along the loop (right) for Material B in Case II.

The final set of experiments concerns the same Material B in Case II. Figure 7 displays load-displacement diagrams and global volume fractions along the loop for different time-steps and different meshes as before. All discretizations indicate the same hysteresis loop and from looking at the figures, one might find support for convergence in time and space for this example.

The adaptive algorithms were run for the Material B as well but did not improve the energy convergence significantly. As there is no theoretical link to the stress field in this incompatible case, we omit further discussion of numerical results.

Acknowledgements. The research of CC was supported by the Max Plank Institute in the Sciences of Leipzig, Germany, and the German Research Foundation through the DFG-Schwerpunktprogramm multi-scale problems. The research of PP was supported in part by grant UDRF-19990115 from University of Delaware Research Foundation and by the German Research Foundation (DFG) as Visiting Professor at the Graduiertenkolleg "Effiziente Algorithmen und Mehrskalenmethoden" in Kiel, Germany. The authors thank A. Mielke for valuable discussions.

\section{REFERENCES}

[1] J.M. Ball and R.D. James, Fine phase mixtures as minimisers of energy. Arch. Rational Mech. Anal. 100 (1987) 13-52.

[2] J.M. Ball and R.D. James, Proposed experimental tests of the theory of fine microstructure and the two-well problem. Philos. Trans. Roy. Soc. London Ser. A 338 (1992) 389-450. 
[3] M. Bildhauer, M. Fuchs and G. Seregin, Local regularity of solutions of variational problems for the equilibrium configuration of an incompressible, multiphase elastic body. Nonlin. Diff. Equations Appl. 8 (2001) 53-81.

[4] S.C. Brenner and L.R. Scott, The mathematical theory of finite element methods, in Texts in Applied Mathematics 15. Springer-Verlag, New York (1994).

[5] C. Carstensen and S. A. Funken, Constants in Clément-interpolation error and residual based a posteriori estimates in finite element methods. East-West J. Numer. Math. 8 (2000) 153-175.

[6] C. Carstensen and S. A. Funken, Fully reliable localised error control in the FEM. SIAM J. Sci. Comput. 21 (2000) $1465-1484$.

[7] C. Carstensen and S. Müller, Local stress regularity in scalar non-convex variational problems. In preparation.

[8] C. Carstensen and P. Plecháč, Numerical solution of the scalar double-well problem allowing microstructure. Math. Comp. 66 (1997) 997-1026.

[9] C. Carstensen and Petr Plecháč, Numerical analysis of compatible phase transitions in elastic solids. SIAM J. Numer. Anal. 37 (2000) 2061-2081.

[10] P.G. Ciarlet, The Finite Element Method for Elliptic Problems. North-Holland Publishing Company, Amsterdam, New York, Oxford (1978).

[11] H. Le Dret and A. Raoult, Variational convergence for nonlinear shell models with directors and related semicontinuity and relaxation results. Arch. Rational Mech. Anal. 154 (1999) 101-134.

[12] K. Eriksson, D. Estep, P. Hansbo and C. Johnson, Introduction to adaptive methods for differential equations, in Acta Numerica, A. Iserles, Ed., Cambridge University Press, Cambridge (1995) 105-158.

[13] I. Fonseca and S. Müller, A-quasiconvexity, lower semicontinuity and Young measures. SIAM J. Math. Anal. 30 (1999) $1355-1390$.

[14] J. Goodman, R.V. Kohn and L. Reyna, Numerical study of a relaxed variational problem from optimal design. Comput. Methods Appl. Mech. Engrg. 57 (1986) 107-127.

[15] A. G. Khachaturyan, Theory of Structural Transformations in Solids. John Wiley \& Sons, New York (1983).

[16] M.S. Kuczma, A. Mielke and E. Stein, Modelling of hysteresis in two-phase systems. Solid Mechanics Conference (1999); Arch. Mech. 51 (1999) 693-715.

[17] R.V. Kohn, The relaxation of a double-well energy. Contin. Mech. Thermodyn. 3 (1991) 193-236.

[18] M. Luskin, On the computation of crystalline microstructure, in Acta Numerica, A. Iserles, Ed., Cambridge University Press, Cambridge (1996) 191-257.

[19] A. Mielke and F. Theil, A mathematical model for rate-independent phase transformations with hysteresis, in Workshop of Continuum Mechanics in Analysis and Engineering, H.-D. Alber, D. Bateau and R. Farwig, Eds. , Shaker-Verlag, Aachen (1999) 117-129.

[20] A. Mielke, F. Theil and V. I. Levitas, A variational formulation of rate-independent phase transformations using an extremum principle. Submitted to Arch. Rational Mech. Anal.

[21] A. L. Roitburd, Martensitic transformation as a typical phase transformation in solids, in Solid State Physics 33, Academic Press, New York (1978) 317-390.

[22] G.A. Seregin, The regularity properties of solutions of variational problems in the theory of phase transitions in an elastic body. St. Petersbg. Math. J. 7 (1996) 979-1003, English translation from Algebra Anal. 7 (1995) 153-187.

[23] R. Verfürth, A review of a posteriori error estimation and adaptive mesh-refinement techniques, in Wiley-Teubner Series Advances in Numerical Mathematics, John Wiley \& Sons, Chichester; Teubner, Stuttgart (1996).

To access this journal online: www.edpsciences.org 